\title{
Tibial Tubercle Avulsion Fractures in Adolescents: A Narrative Review \& Case Report
}

\author{
Gregory W. Kunis ${ }^{1 *}$, Joshua A. Berko', Jeffrey C. Shogan', Joshua B. Sharan', Derek Jones ${ }^{2}$ \\ 'Nova Southeastern University, Kiran C. Patel College of Osteopathic Medicine, Florida, USA \\ ${ }^{2}$ Department of Orthopedic Surgery, Broward Health, Florida, USA
}

\section{Article Info}

\section{Article Notes}

Received: September 06, 2021

Accepted: October 26, 2021

\section{${ }^{*}$ Correspondence:}

Mr. Gregory W. Kunis, Nova Southeastern University, Kiran C.

Patel College of Osteopathic Medicine, Florida, USA;

Email: gk335@mynsu.nova.edu

${ }^{0} 2021$ Kunis GW. This article is distributed under the terms of the Creative Commons Attribution 4.0 International License.

\section{Keywords:}

Tibial tubercle

Avulsion fracture

Tibial tuberosity

Ogden classification

Watson-Jones

Patellar tendon rupture

\section{Abstract}

Intro: Tibial tuberosity avulsion fractures are rare fracture patterns accounting for less than $1 \%$ of all pediatric fractures. These fractures occur when there is a sudden unbalancing of forces through the patellar tendon that separates the tibial tubercle from the anterior portion of the proximal tibia. These forces are commonly introduced in sporting activities and show a predominance for adolescent males. Treatment with open reduction internal fixation commonly results in favorable outcomes with minimal complications. In this presentation, we explore a case of a tibial tuberosity avulsion fracture and give an in-depth review of all aspects concerning this fracture pattern.

Case Description: A 14-year-old male with no significant past medical history presented via emergency medical services after a ground level fall while playing basketball. Radiographs of the left knee and tibia revealed an Ogden Type III, distracted avulsion fracture of the tibial tuberosity with suprapatellar effusion. Surgical intervention was achieved through open reduction internal fixation of the left tibial tubercle.

Discussion: Although a relatively rare fracture pattern, this case demonstrates a classic presentation and treatment of a tibial tuberosity avulsion fracture. This case serves as a reminder that despite the rarity of the injury, a clinician with an appropriate index of suspicion can accurately diagnose and treat this fracture and achieve positive outcomes in returning the patient to pre-injury activities. For those reasons, we provide a comprehensive overview of all aspects regarding this fracture pattern including the anatomy, embryology, mechanism of action, predisposing conditions, treatment considerations, complications and associated injuries.

\section{Introduction}

\section{Incidence}

Tibial tuberosity avulsion fractures are extremely rare fracture patterns, with reported incidence rates of $0.4 \%$ to $2.7 \%$ of all epiphyseal injuries, $<1 \%$ of all physeal injuries, and about $3 \%$ of all proximal tibial injuries ${ }^{1,2,3}$. This injury pattern accounts for less than $1 \%$ of all pediatric fractures and is even less common in adult populations.

\footnotetext{
Anatomy

The tibial tubercle is a bony prominence on the midline anterior tibia just inferior to where the anterior surfaces of the medial and lateral tibial condyles join. The tibial tubercle forms in four separate stages as described by Ehrenborg ${ }^{4,5,6}$. The first stage is the cartilaginous stage which begins by the 15 th week of gestation ${ }^{7}$ and is succeeded by the apophyseal stage in which the tibial tubercle's secondary ossification center becomes apparent. This stage begins
} 
in females between the ages of 8 to 12 years old and in males from 9 to 14 years old ${ }^{4}$. The epiphyseal stage follows with merging of the apophysis and the epiphysis of the proximal tibia. This stage occurs in females between the ages of 10 to 15 years old and in males from 11 to 17 years old ${ }^{4}$. Following the epiphyseal stage is the final stage resulting in bony fusion which completes the formation process in females by age 15 and males by age $17^{4}$.

In addition to the different stages of bony development, there are microscopic changes that also predispose to tibial tubercle avulsion fractures. Ogden et al. ${ }^{7,8}$ analyzed the tibial tubercle growth plate and described three histological zones. Preceding closure of the growth plate, there is a progressive change from fibrocartilage to columnar cartilage transitioning proximal to distal. The physiologic epiphysiodesis follows beginning at the proximal tibial physis progresses distally. These histological changes predispose the tibial tubercle to avulsion in the later stages of closure ${ }^{9,10}$. Figure 1 shows the primary ossification site at the proximal tibial physis and the secondary ossification site distally underneath the tibial tubercle.

\section{Mechanism of Action}

The mechanism of tibial tubercle avulsion fractures surrounds the extensor mechanism of the knee and is strongly associated with jumping and landing. The two proposed mechanisms of injury are a strong quadriceps contraction during knee extension, as in a jumping movement, or a passive flexion of the knee while the quadriceps is contracting, as in a landing movement $t^{1,4}$. Galos et al. ${ }^{11}$ described the function of the patella in the extensor mechanism as, "a fulcrum to increase efficiency and the rotational moment provided by the pull of the quadriceps by moving the lever arm farther away from the center of rotation of the knee joint." This increased force on the tibial tubercle exceeds the strength of the physis, the perichondrium, and the periosteum adjacent to the tubercle

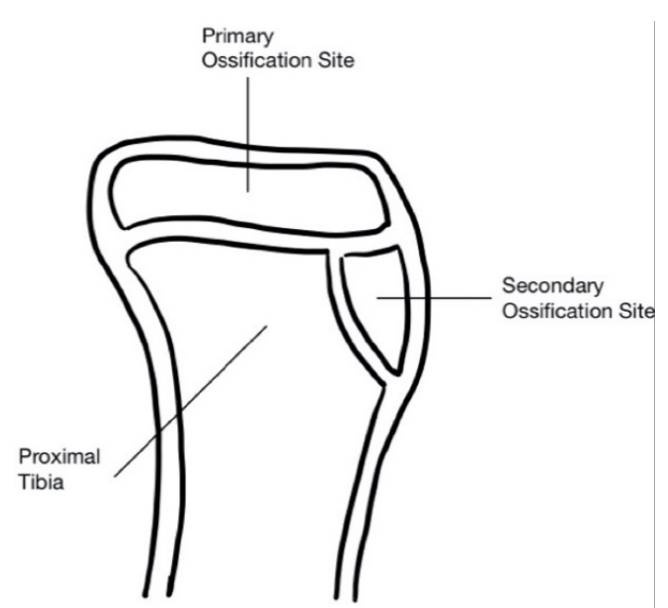

Figure 1: Anatomy of tibial physes and ossification sites due to the inherent instability of this area in adolescents since the physis has yet to close ${ }^{4,12}$. Gao et al. ${ }^{13}$ described the variability of extensor mechanism's failure as one of five types: avulsion at subchondral bone, avulsion at the cement line, failure through calcified fibrocartilage, tearing through uncalcified fibrocartilage, and rupture through the mid-substance of the ligament. The variability in extensor mechanism failure as well as in the fracture pattern are correlated to the extent of physeal closure when the injury occurs $^{14}$.

In a systematic review, Pretell-Mazzini et al. ${ }^{14}$ found that this injury is more common in the left knee $(59 \%)$ and in males $(97 \%)$ as compared to females. This is attributed to the higher number of males participating in high impact sports, the inherently increased strength of the quadriceps muscles in men as compared to women, and the later age of closure of the physis in males ${ }^{4,15,16,17,18}$. These factors compound on one another and provide an explanation for the failure of the extensor mechanism seen in this injury. Although this combination of factors is that which is seen most commonly, there have been reports of tibial tubercle avulsion fractures seen in low-impact sports such as running $g^{4,19,20,21}$. These reports are likely due to a host of other factors, predisposing for weakness at the physeal line.

One study looked at the relationship between body mass index (BMI) and these low-impact tibial tubercle avulsion fractures, but found no significant correlation for this to be considered a risk factor in the setting of no trauma ${ }^{6}$. Although BMI was not found to be a risk factor, there are a host of predisposing conditions that may lead to an even higher incidence of tibial tubercle avulsion fractures.

\section{Predisposing Conditions}

Aside from a traumatic imbalance of mechanical load that overpowers the healthy anatomy, there are several conditions that have been described in the literature that may play a role in directly weakening the tibial tuberosity or causing abnormal loading of the anatomy and predisposing its fracture. These include Osgood-Schlatter disease (OSD), osteogenesis imperfecta, Sinding-Larsen Johanssen (SLJ) Syndrome, patella baja, tight hamstrings, and other physeal anomalies with OSD being the most extensively documented in the literature ${ }^{16,20,22,23}$. OSD has previously been indicated as a predisposing factor, however no formal association has been established due to many cases having confounding, unrelated comorbidities ${ }^{1,4,15,24,25,26}$.

Pesl and Havranek ${ }^{10}$ described several studies of acute tibial tubercle injuries in patient populations with OSD. Ogden et al. ${ }^{27}$ documented nine of 14 patients with acute injuries as having pre-existing OSD leading him to suggest a possible predispositional relationship. Ogden suggests that OSD may alter the histology of the tissue which could in 

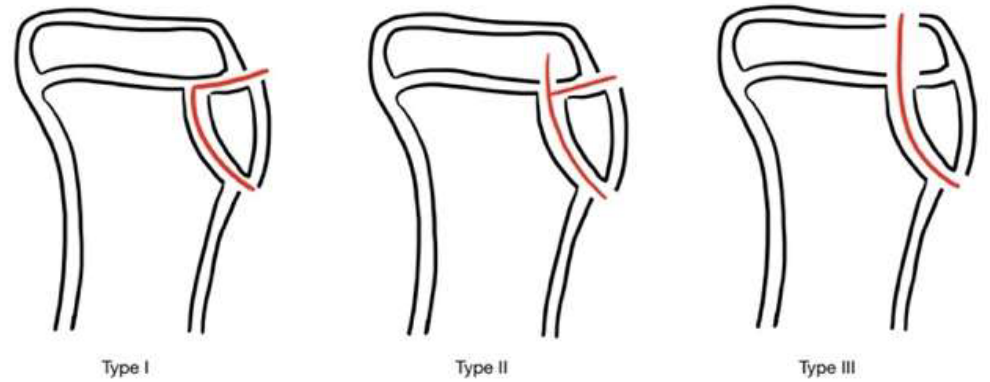

Figure 2: Watson-Jones Classification System
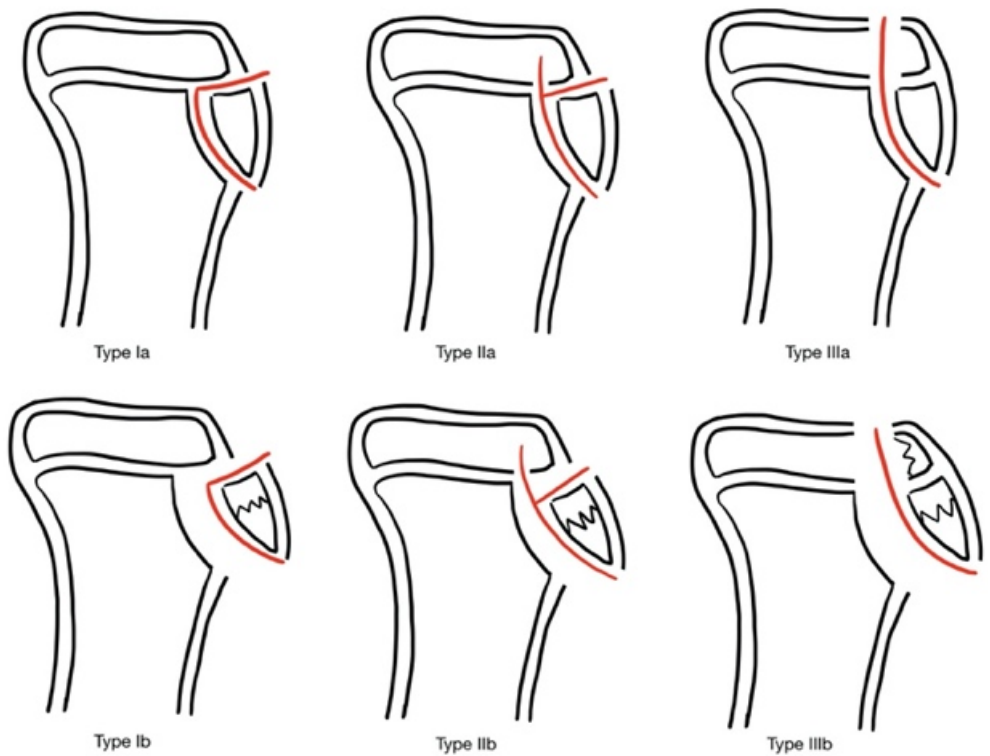

Figure 3: Ogden Classification System

turn alter its biomechanical properties and predispose it to avulsion fractures. He further suggested that microscopic changes in the histology caused by OSD may affect the properties of the surrounding tissue, thus predisposing the tibial tubercle to avulse. Other studies outlined by Pesl and Havranek ${ }^{10}$ that have a record of OSD present in their patient populations include Bolesta and Fitch ${ }^{1}$ with four of 16 patients, Mosier and Stanitski ${ }^{9}$ two of 19 , Pesl and Havranek ${ }^{10}$ one of 12 , and Chow et al. ${ }^{15}$ one of 16 patients.

Bradko et al. ${ }^{22}$ described a case of a 12 year old male with OSD experiencing a tibial tubercle avulsion fracture during a basketball game. They postulated that traction apophysitis diseases, namely OSD and SLJ Syndrome may predispose to avulsion style fractures. OSD occurring from repetitive traction at the tibial tubercle typically occurs in male athletes between the ages of 10-15 22 . SLJ Syndrome on the other hand, is a less common etiology in which irritation of the growth plate occurs at the inferior portion of the patella in younger patients ages 10-12 years old ${ }^{22}$. This literature indicates that clinicians should exhibit a high index of suspicion in patient populations with these pre-existing conditions, however, a formal causal relationship has not yet been established.

\section{Classification}

Several different classification schemes exist that have been proposed and modified over the years. WatsonJones ${ }^{28}$ proposed the initial classification scheme in 1955 and outlined three different types of tibial tuberosity fracture patterns based on location and involvement. Type I is any tubercle avulsion distal to the physis, or rather a fracture of the distal tibial tuberosity. A Type II fracture has some involvement in the physis, however, the knee joint is spared from injury. Lastly, a Type III is an extension of the fracture into the joint space. Figure 2 shows the WatsonJones classification system: Types I, II, and III.

This classification scheme received its first expansion in 1980 by Ogden ${ }^{27}$ who added designations to further specify fracture displacement and comminution of each type. Ogden's "A" classification bodes for nondisplaced fractures and "B" indicates displaced or comminuted fractures. Figure 3 shows the Ogden classification system: Types Ia, IIa, IIIa, Ib, IIb, and IIIb.

The next modification was made by Ryu and Debenham ${ }^{29}$ who suggested a Type IV designation in addition to the 
original three types proposed by Watson-Jones. A Type IV classification would be reserved for avulsion fractures that move posterior through the physis with the potential to displace the entire physis and tubercle, or plainly put, an avulsion fracture of the entire proximal tibial epiphysis.

In 1990 Frank et al. ${ }^{30}$ published a case report in which they outlined a group $\mathrm{C}$ modification reserved for fractures with patellar ligament avulsion. Later, McKoy and Sitanksi ${ }^{4}$ suggested a Type $\mathrm{V}$ fracture to follow a Ryu Type IV. A Type $\mathrm{V}$ fracture is the joining of a Type IIIb (displaced intraarticular) and a Type IV (posterior translation) which results in a "Y" configuration. Figure 4 shows the Ogden classification addition by Ryu and Debenham: Type IV. Figure 5 shows the Ogden classification addition by Frank et al.: Type V.

Lastly, in 2012, Pandya ${ }^{12}$ proposed a new four-tier classification system altogether based on 3D geometry and complex patterns of physeal closure. Type A designates an

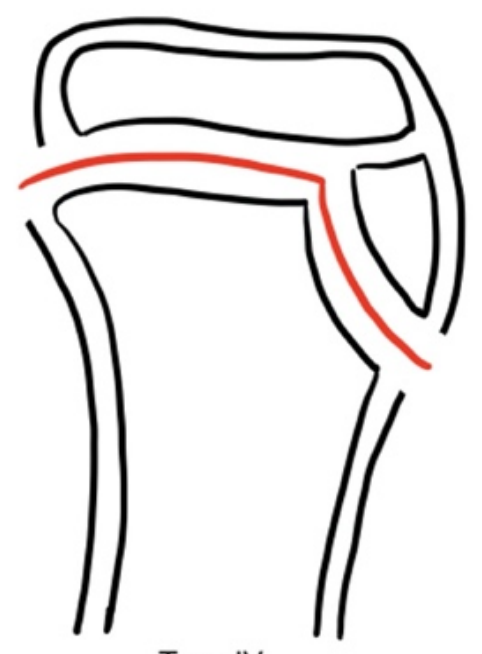

Type IV

Figure 4: Ryu \& Debenham addition

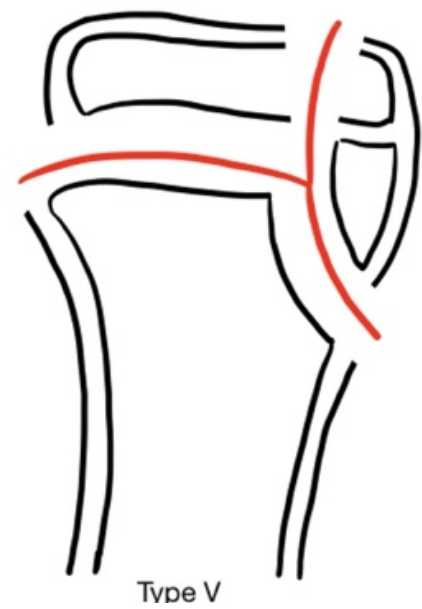

Figure 5: Frank et al. addition

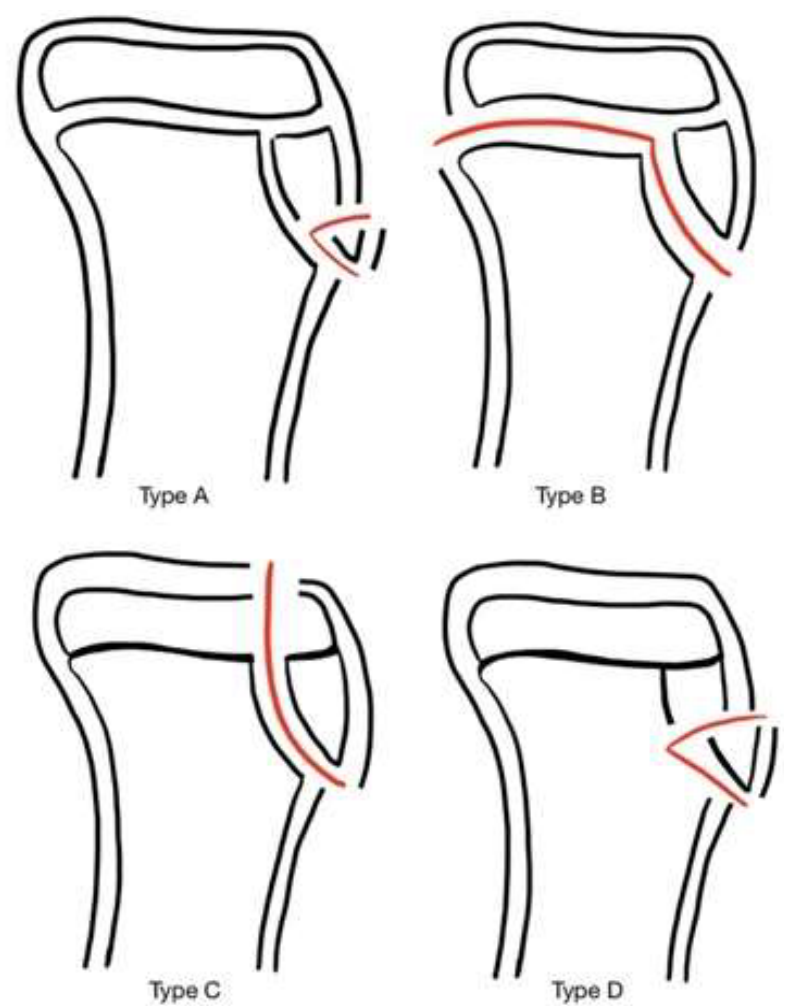

Figure 6: Pandya Classification System

isolated fracture with an ossified tip. Type B is an epiphysis and tubercle fracture from the metaphysis without intraarticular involvement. Type $\mathrm{C}$ is an extension of a Type 2 fracture with intra-articular involvement and extension into the proximal tibia, with the differentiating factor being that the physis is closed. A Type D involves the distal tubercle, with again the physis being closed. Figure 6 shows the Pandya classification: Types, A, B, C, and D. Despite the many modifications made to the original Watson-Jones criteria, the most popularly used is the Ogden modification. The others can be reserved for highly specific cases.

\section{Case Report}

A 14-year-old male with no significant past medical history presented via emergency medical services after a ground level fall while playing basketball. The patient states he was playing basketball when he jumped up and then landed with his left knee in a flexed position. He immediately felt a pop in his left knee as well as immediate pain and swelling about the left knee. The left lower extremity was placed in a long leg posterior splint in the emergency department. The patient was able to move his toes and all sensation was intact, as were pedal pulses, demonstrating he remained neurovascularly intact. Radiographs of the left knee and tibia revealed an Ogden Type IIIb, distracted avulsion fracture of the tibial tuberosity with suprapatellar joint effusion. Figure 7 shows the preoperative x-rays.

The patient was subsequently taken to the operating 

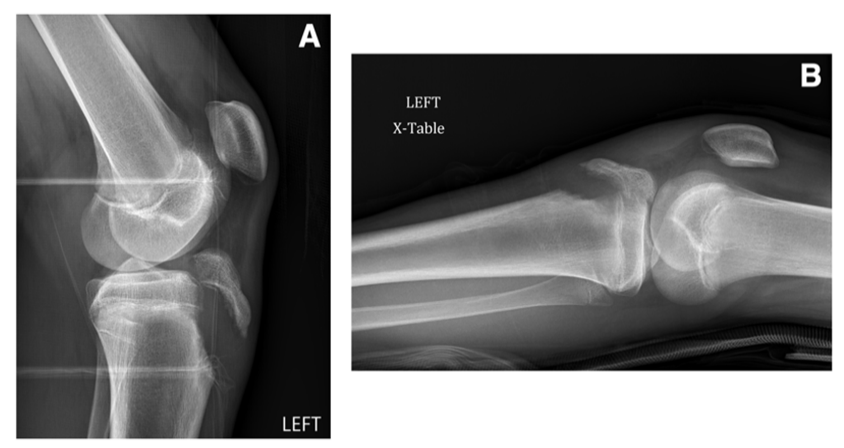

Figure 7: Pre-op x-rays; (A) shows a lateral view; (B) shows a crosstable view
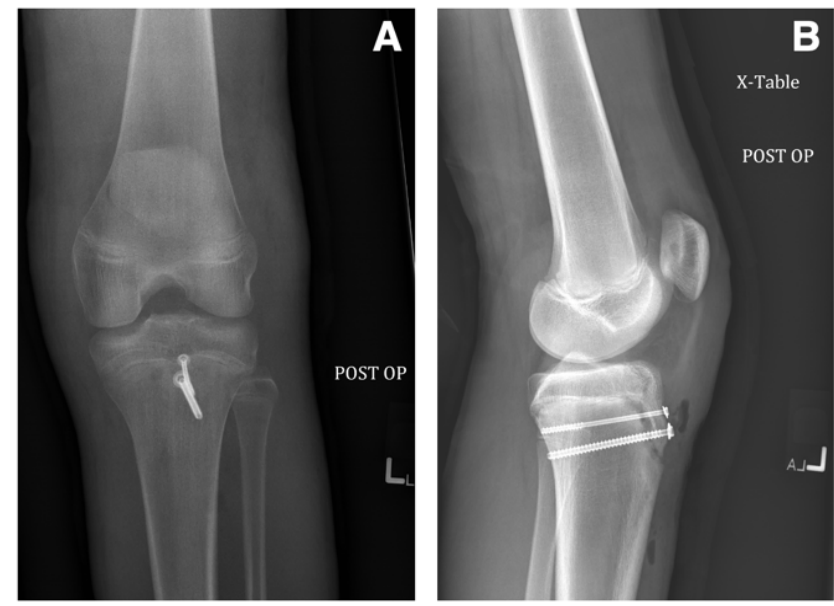

Figure 8: Post-op x-rays; (A) shows an AP view; (B) shows a crosstable lateral view

room and surgical intervention was achieved through open reduction internal fixation of the left tibial tubercle. A $4 \mathrm{~cm}$ incision was made over the tibial tubercle. The fascia was dissected and the fracture hematoma was evacuated from the knee joint using a sterile syringe. Approximately $70 \mathrm{cc}$ of hematoma was evacuated. The fracture was identified and debrided and copiously irrigated. It was reduced using direct pressure from an elevator and held in place with a skin rake. The threaded guide pin for the 4.0 partiallythreaded cancellous screw was inserted from the anteriorto-posterior direction. Biplanar fluoroscopy measurements were taken off of the screw and the screw was inserted over the guidewire and the guidewire was then removed. A more distal pin site was then selected and the partially threaded guidewire for the 5.0 cannulated screw was then drilled and placed and checked under biplane fluoroscopy. The 5.0 fully-threaded cannulated screw was then inserted over the guidewire which was then removed. Proper positioning was confirmed on both AP and lateral views and the fracture was securely fixed. The fracture was found to have disrupted the anterior tibial fascial plane of the tibialis anterior. This was sharply debrided and then tacked down in place. The patient was weight-bearing as tolerated to the operative extremity while in a knee immobilizer, and checked for any signs of compartment syndrome regularly. The patient had no complications and was discharged three days post-op. Figure 8 shows the postoperative $\mathrm{x}$-rays.

This patient's post-operative course was uncomplicated and he returned to normal activity within four months, after completing physical therapy. The patient had no deficits at this time and regained full function and range of motion. No leg-length discrepancy was noted and no complications were present at any point throughout the post-operative recovery and rehabilitation period. There was no further imaging done at this time, however the patient had no complaints and seemed to be back to his previous baseline. Interestingly, this patient came back a year later for the same injury to the right side which also required surgical fixation. This suggests the possibility of underlying anatomical abnormalities predisposing to this specific injury, however none of the previously described abnormalities were apparent in this case.

\section{Discussion}

\section{Associated Injuries/Complications}

Tibial tubercle avulsion fractures are associated with a wide variety of additional injuries and the repair has shown a few common complications. Most of the complications occur with higher-grade or intra-articular fractures. Patellar tendon rupture, quadriceps tendon avulsion, meniscal tears, cruciate laxity, and compartment syndrome were shown to be the most commonly reported associated injuries $^{12,14,31,32,33}$. In one study that comprised of 336 tibial tubercle avulsion fractures in adolescent patients, these concomitant injuries were shown at rates of $(2 \%)$ patellar or quadriceps tendon avulsions, $(2 \%)$ meniscal tears, $(1 \%)$ increased ligamentous laxity, and (4\%) compartment syndromes ${ }^{14}$. There was not a significant correlation found between meniscus tears and any particular fracture type, however all tears were seen in Type III fractures ${ }^{14}$, therefore it is suggested that a pre-operative MRI be done to evaluate the meniscus in Type III and Type IV intraarticular fractures.

Preoperative compartment syndrome has been associated with Type II and Type IV fractures, but only scarcely in the literature ${ }^{34,35}$. Post-op, the most common complication has been shown to be bursitis in $56 \%$ of patients ${ }^{14,34}$, of which only $7 \%$ required implant removal. The most devastating post-op complication has been compartment syndrome, arising from soft tissue injury or injury to the anterior tibial recurrent artery, a branch of the anterior tibial artery that is located adjacent to the lateral border of the tibial tubercle ${ }^{16,34,36,37}$. This complication has been seen in $10-20 \%$ of cases in the literature ${ }^{12,32,35}$.

Other complications that have been reported are 
refracture, malunion, nonunion, infection, saphenous neuroma, genu recurvatum, and limb-length discrepancy ${ }^{1,10,14,15,27,29,38}$. Genu recurvatum has been associated with patients under 11 years of age because of premature closure of the anterior physis ${ }^{4}$, and in a systematic review by Pretell-Mazini et al. ${ }^{14}$ this sequela was seen in $4 \%$ of patients, all were patients younger than 13 years of age. This study also reported limb-length discrepancy that has been seen in $5 \%$ of patients, with a mean discrepancy of $1.4 \mathrm{~cm}$. As far as neurological sequelae, saphenous nerve neuroma has been reported ${ }^{1}$, as well as some numbness over the tibial tuberosity ${ }^{14}$.

\section{Treatment Considerations}

Ogden Type Ia injuries have been treated conservatively with plaster casting for several weeks and immobilization with excellent results. Closedreduction with internal-fixation remains a treatment modality that can be considered for the intra-articular form of a tibial avulsion (Types IIIa and IIIb). Openreduction with internal-fixation can also be used for displaced intra-articular variants. While there seems to be some latitude regarding the approach the clinician may take in treating these injury types (Ia, IIIa, and IIIb), it is recommended by Pesl and Havranek ${ }^{10}$ that openreduction with internal-fixation is the only appropriate treatment for the extra-articular form of the injury (Ib and IIa) due to either large displacement of a small tibial tubercle fragment or intervening soft tissue making closed-reduction unfeasible.

It is important to consider the age of the patient in selecting the appropriate treatment modality. Of course, proper reduction must be achieved and maintained against the superiorly directed pull of the quadriceps, but maintenance of this reduction can potentially compromise skeletal growth considering the proximity of the physis. Fortunately, many patients who present with this injury are near the end of cartilaginous growth - usually male adolescents between ages 13 and 16 .

The superiorly directed pull of the quadriceps tendon on the patella can also introduce gaps in the repair which serve as an impediment to proper healing. Considering this, perhaps the most widely used technique for the patellar tendon repair in a tibial avulsion is to use \#2 nonabsorbable sutures in a Krackow type pattern. The Krackow type pattern has demonstrated superior resistance to gap formation as well as increased load required for repair failure compared to a three-loop pulley suture in Achilles tendon repair in canine models ${ }^{39}$. In the case of a bifocal tibial avulsion, Fiber Wire and cortical screws maintained adequate reduction while allowing early range of motion in at least one $\operatorname{case}^{40}$.

\section{Outcomes}

Overall, post-operative protocols in the existing literature show a predilection for immobilization in the immediate post-operative period, early weight-bearing, and early range of motion. These protocols generally mange pain symptomatically and have the patient follow-up with physical therapy for strength and stability rehabilitation. Behery et al. ${ }^{40}$ prefer a knee immobilizer for the first week during full weight-bearing, and early range of motion with restriction to $60^{\circ}$ of flexion to protect the repair from excessive tensile stress. Range of motion is usually advanced at the discretion of the individual surgeon, pending followup imaging demonstrating adequate bone healing as well as sufficient advancement in physical therapy. PretellMazzini et al. ${ }^{14}$ found in their systematic review that $98 \%$ of patients achieved full range of knee motion by 22.3 weeks on average, and $94 \%$ percent were able to return to preinjury activities by 28.9 weeks on average. They also showed that there was no statistically significant difference among fracture types in regard to return to preinjury activities or range of motion, with fracture union reported in all 23 articles with a $99 \%$ healing rate.

\section{Conclusion}

Although a relatively rare fracture pattern, this case demonstrates a classic presentation and treatment of a tibial tuberosity avulsion fracture. This case serves as a reminder that despite the rarity of the injury, a clinician with an appropriate index of suspicion can accurately diagnose and treat this exceptional fracture and achieve positive outcomes in returning the patient to pre-injury activities. Being able to identify and classify the fracture allows for determination of fixation method as well as raising awareness to possible complications. Knowledge of predisposing conditions as well as associated injuries allows for a more comprehensive understanding of this injury, and ultimately better management and outcomes. With this review, clinicians should have a complete understanding of tibial tuberosity avulsion fractures leading to enhanced patient care and satisfaction.

\section{Acknowledgement}

The production of this manuscript as well as the research within was conducted independently without any financial support.

\section{Conflict of Interest}

The authors have no conflicts of interest to declare. Each co-author has reviewed the contents of the completed manuscript and agree with it. There are no financial interests to report.

\section{References}

1. Bolesta MJ, Fitch RD. Tibial tubercle avulsions. J Pediatr Orthop. 1986; 6(2): 186-92. 
2. Shelton WR, Canale ST. Fractures of the tibia through the proximal tibial epiphyseal cartilage. J Bone Joint Surg Am. 1979; 61(2): 167-73.

3. Hand WL, Hand CR, Dunn AW. Avulsion fractures of the tibial tubercle. J Bone Joint Surg Am. 1971; 53(8): 1579-83.

4. McKoy BE, Stanitski CL. Acute tibial tubercle avulsion fractures. Orthop Clin North Am. 2003; 34(3): 397-403.

5. Ehrenborg G, Engfeldt B. The insertion of the ligamentum patellae on the tibial tuberosity. Some views in connection with the OsgoodSchlatter lesion. Acta Chir Scand. 1961; 121: 491-9.

6. Shin Y-W, Kim D-W, Park K-B. Tibial tubercle avulsion fracture according to different mechanisms of injury in adolescents: Tibial tubercle avulsion fracture: Tibial tubercle avulsion fracture. Medicine (Baltimore). 2019; 98(32): e16700.

7. Ogden JA, Hempton RJ, Southwick WO. Development of the tibial tuberosity. Anat Rec. 1975; 182(4): 431-45.

8. Ogden JA. Radiology of postnatal skeletal development. X. Patella and tibial tuberosity. Skeletal Radiol. 1984; 11(4): 246-57.

9. Mosier SM, Stanitski CL. Acute tibial tubercle avulsion fractures. Pediatr Orthop. 2004; 24(2): 181-4.

10. Pesl T, Havranek P. Acute tibial tubercle avulsion fractures in children selective use of the closed reduction and internal fixation method. Child Orthop. 2008; 2(5): 353-6.

11. Galos DK, Konda SR, Kaplan DJ, et al. Transosseous-equivalent repair for distal patellar tendon avulsion. Arthrosc Tech. 2016; 5(2): e385-9.

12. Pandya NK, Edmonds EW, Roocroft JH, et al. Tibial tubercle fractures: complications, classification, and the need for intra-articular assessment. J Pediatr Orthop. 2012; 32(8): 749-59.

13. Gao J, Räsänen T, Persliden J, et al. The morphology of ligament insertions after failure at low strain velocity: an evaluation of ligament entheses in the rabbit knee. J Anat. 1996; 189(Pt 1): 127-33.

14. Pretell-Mazzini J, Kelly DM, Sawyer JR, et al. Outcomes and complications of tibial tubercle fractures in pediatric patients: A systematic review of the literature. J Pediatr Orthop. 2016; 36(5): 440-6.

15. Chow SP, Lam JJ, Leong JC. Fracture of the tibial tubercle in the adolescent. J Bone Joint Surg Br. 1990; 72(2): 231-4.

16. Jakoi A, Freidl M, Old A, et al. Tibial tubercle avulsion fractures in adolescent basketball players. Orthopedics. 2012; 35(8): 692-6.

17. Silva Júnior AT da, Silva LJ da, Silva Filho UC da, et al. Anterior avulsion fracture of the tibial tuberosity in adolescents - Two case reports. Rev Bras Ortop. 2016; 51(5): 610-3.

18. Zrig M, Annabi H, Ammari T, et al. Acute tibial tubercle avulsion fractures in the sporting adolescent. Arch Orthop Trauma Surg. 2008; 128(12): 1437-42.

19. Özokyay L, Michler K, Müsgens J. Beidseitige atraumatische tibiakopfepiphysiolyse. Unfallchirurg. 2002; 105: 735-9.

20. Nicolini AP, Carvalho RT, Ferretti M, et al. Simultaneous bilateral tibial tubercle avulsion fracture in a male teenager: case report and literature review. J Pediatr Orthop B. 2018; 27(1): 40-6.

21. Khoriati A-A, Guo S, Thakrar R, et al. Bilateral atraumatic tibial tubercle avulsion fractures: case report and review of the literature. Injury. 2015; 46(4): 767-9.
22. Bradko V, Stoll WT, Haruno LS, et al. Two cases of combined patellar tendon avulsion from the tibia and patella. SICOT J. 2018; 4: 17.

23. Roy SP, Nag K. Simultaneous bilateral tibial tuberosity avulsion fractures in adolescence: case report and review of 60 years of literature. Injury. 2013; 44(12): 1953-5.

24. Levi JH, Coleman CR. Fracture of the tibial tubercle. Am J Sports Med. 1976; 4(6): 254-63.

25. Christie MJ, Dvonch VM. Tibial tuberosity avulsion fracture in adolescents. J Pediatr Orthop. 1981; 1(4): 391-4.

26. Lepse PS, Mccarthy RE, Mccullough FL. Simultaneous bilateral avulsion fracture of the tibial tuberosity: A case report. Clin Orthop Relat Res. 1988.

27. Ogden JA, Tross RB, Murphy MJ. Fractures of the tibial tuberosity in adolescents. J Bone Joint Surg Am. 1980; 62(2): 205-15.

28. Rogers WA. Fractures and joint injuries, vol. 2, ed. 4. Sir Reginald Watson-Jones, B.sc., M.ch.Orth., F.r.c.s., F.r.a.c.s. (hon.), F.a.c.s. (hon.). Edinburgh, E. \& S. livingstone, ltd.; Baltimore, the Williams and Wilkins company, 1955. \$22.50 (two volumes). J Bone Joint Surg Am. 1955; $37(6): 1326$

29. Ryu RK, Debenham J0. An unusual avulsion fracture of the proximal tibial epiphysis. Case report and proposed addition to the WatsonJones classification. Clin Orthop Relat Res. 1985; (194): 181-4.

30. Frankl U, Wasilewski SA, Healy WL. Avulsion fracture of the tibial tubercle with avulsion of the patellar ligament. Report of two cases. J Bone Joint Surg Am. 1990; 72(9): 1411-3.

31. Clarke DO, Franklin SA, Wright DE. Avulsion fracture of the tibial tubercle associated with patellar tendon avulsion. Orthopedics. 2016; 39(3): e561-4.

32. Frey S, Hosalkar H, Cameron DB, et al. Tibial tuberosity fractures in adolescents. J Child Orthop. 2008; 2(6): 469-74.

33. Wiss DA, Schilz JL, Zionts L. Type III fractures of the tibial tubercle in adolescents. J Orthop Trauma. 1991; 5(4): 475-9.

34. Brey JM, Conoley J, Canale ST, et al. Tibial tuberosity fractures in adolescents: is a posterior metaphyseal fracture component a predictor of complications? J Pediatr Orthop. 2012; 32(6): 561-6.

35. Polakoff DR, Bucholz RW, Ogden JA. Tension band wiring of displaced tibial tuberosity fractures in adolescents. Clin Orthop Relat Res. 1986; (209): 161-5.

36. Wall JJ. Compartment syndrome as a complication of the Hauser procedure. J Bone Joint Surg Am. 1979; 61(2): 185-91.

37. Cummings RJ, Webb HW, Lovell WW, et al. The popliteal artery entrapment syndrome in children. J Pediatr Orthop. 1992; 12(4): $539-41$.

38. Albuquerque RP e., Giordano V, Carvalho ACP, et al. Fratura avulsão bilateral e simultânea da tuberosidade tibial em uma adolescente: relato de caso e terapêutica adotada. Rev Bras Ortop. 2012; 47(3): 381-3.

39. Wilson L, Banks T, Luckman P, et al. Biomechanical evaluation of double Krackow sutures versus the three-loop pulley suture in a canine gastrocnemius tendon avulsion model. Aust Vet J. 2014; 92(11): 427-32.

40. Behery OA, Feder OI, Beutel BG, et al. Combined tibial tubercle fracture and patellar tendon avulsion: Surgical technique and case report. J Orthop Case Rep. 2018; 8(3): 18-22. 